\title{
A PRODUCTIVITY EVALUATION MODEL BASED ON INPUT AND OUTPUT ORIENTATIONS
}

\author{
C.O. Anyaeche ${ }^{1}$ and A.E.Oluleye ${ }^{2}$ \\ ${ }^{1}$ Department of Industrial and Production Engineering \\ University of Ibadan, Nigeria \\ ${ }^{1}$ osita.anyaeche@mail.ui.edu.ng, ${ }^{2}$ ayodeji.oluleye@mail.ui.edu.ng
}

\begin{abstract}
Many productivity models evaluate either the input or the output performances using standalone techniques. This sometimes gives divergent views of the same system's results. The work reported in this article, which simultaneously evaluated productivity from both orientations, was applied on real life data. The results showed losses in productivity $(-2 \%)$ and price recovery $(-8 \%)$ for the outputs; the inputs showed productivity gain $(145 \%)$ but price recovery loss $(-63 \%)$. These imply losses in product performances but a productivity gain in inputs. The loss in the price recovery of inputs indicates a problem in the pricing policy. This model is applicable in product diversification.
\end{abstract}

\section{OPSOMMING}

Die meeste produktiwiteitsmodelle evalueer of die inset- of die uitsetverrigting deur gebruik te maak van geïsoleerde tegnieke. Dit lei soms tot uiteenlopende perspektiewe van dieselfde sisteem se verrigting. Hierdie artikel evalueer verrigting uit beide perspektiewe en gebruik ware data. Die resultate toon ' $n$ afname in produktiwiteit $(-2 \%)$ en prysherwinning $(-8 \%)$ vir die uitsette. Die insette toon ' $n$ toename in produktiwiteit $(145 \%)$, maar ' $n$ afname in prysherwinning (-63\%). Dit impliseer ' $\mathrm{n}$ afname in produkverrigting, maar ' $\mathrm{n}$ produktiwiteitstoename in insette. Die afname in die prysherwinning van insette dui op ' $\mathrm{n}$ problem in die prysvasstellingbeleid. Hierdie model is geskik vir produkdiversifikasie. 


\section{INTRODUCTION}

Productivity is the ratio between the outputs and the inputs consumed to produce the products marketed. Many productivity models evaluate input or output performance measures using stand-alone techniques. Input-oriented studies such as Swaim and Sink [13], Grossman [6], Hayzen and Reeve [7], are based on the assumption that a given demand has been fulfilled with the gradual reduction in inputs. Output-oriented models are used in certain processes where the input is given and the output is the focus (see Sumanth [12], Prasad [9], and Agrell and Wikner [1]). Focusing on the performance of inputs alone (without simultaneously looking at that of outputs) does not present the total picture (Chan [5], Anyaeche [2]), and could lead to wrong conclusions and decisions (Anyaeche [2]). Productivity is a relative term, which cannot be said to increase or decrease unless a comparison is made (Tangen [14]).

The multi-factor productivity models, including the American Productivity Center (APC) model (Sumanth [13], Grossman [6], Rao [11], Swaim and Sink [13], National Productivity Centre (NPC) [8], Hayzen and Reeve [7]) address profitability, productivity and price recovery factors; however, they do not give the performance measures of the outputs. In particular, the APC model defines the above factors and gives the dynamic measures, but these also are limited to the inputs. A simultaneous evaluation of both the input and the output performances would give two different orientations, making it possible to intervene in both the product line and input management.

\section{MODEL DEVELOPMENT}

Productivity is related to the use and availability of resources. Thus, it is reduced if a company's resources are idle, or if they are not properly used, or if there is a lack of them. It is also strongly linked to the creation of value (Tangen [14]). Thus high productivity is achieved when activities and resources in manufacturing and transformation processes add value to the production process. In this context, production encompasses both manufacturing processes and service delivery.

Any analysis of productivity should therefore recognize that productivity is a ratio, and its evaluation should give the performance of the inputs as well as the outputs. A simultaneous evaluation from both orientations would give an all-inclusive picture.

\subsection{System leakages}

The total conversion costs, which involve both the production and the idle resource costs (Prokopenko [10]), break down production costs into productive, ancillary, and idle components. This work considers non-productive resources as being debited to throughput as leakage. Using the notations in Appendix 1, the total cost of inputs is given by: $\sum_{i=1}^{m} q_{i j k i j}$;

thus if there are no leakages in the production process, the total cost of inputs and the total cost of outputs should be equal - i.e.

$$
\sum_{i=1}^{m} q_{i j} k_{i j}=\sum_{x=1}^{n} t_{x j} u_{x j}
$$

However, if there are leakages, then $\sum_{i=1}^{m} q_{i j} k_{i j}=\sum_{x=1}^{n} t_{x j} U_{x j}+$ leakages.

We can then express the throughput cost as:

$$
\sum_{x=1}^{n} t_{x j} u_{x j}=\sum_{i=1}^{m} q_{i j} k_{i j}-\text { Leakages }
$$


The import of equation (2) is that the total cost of inputs used in production includes the productive (output) and leakage components. In a sense, equation (1) implies that the leakage is zero. Productivity is enhanced if leakage is properly tracked and effectively reduced. To obtain the productivity, price recovery, and profitability factors for output orientation, this work assumes no system leakages. Elsewhere, Anyaeche [2] and Anyaeche and Oluleye [4] highlight system leakage, but do not use the output orientation.

This work uses the APC model's approach for the input resources; however, it extends the performance measures to the outputs values. These are presented in the following sections.

\subsection{Profitability of output resource}

In this section we consider the profitability, productivity, and price recovery factors and their contributions to profit. In a multi-input, multi-output situation, the cost of production viewed from the input orientation is given by $\sum_{i=1}^{m} q_{i j} k_{i j}$, while that from the output orientation is given by: $\sum_{x=1}^{n} t_{x j} U_{x j}$.

Since no leakage is assumed, then: $\sum_{x=1}^{n} t_{x j} u_{x j}=\sum_{i=1}^{m} q_{i j} k_{i j}$

Therefore, the total profitability in period $j, P_{f j}$, can be written as:

$P_{f j}=\sum_{x=1}^{n} t_{x j} c_{x j} / \sum_{x=1}^{n} t_{x j} u_{x j}$

Define the total profitability in period 1 (base period) as $P_{f 1}$, thus:

$P_{f 1}=\sum_{x=1}^{n} t_{x 1} c_{x 1} / \sum_{x=1}^{n} t_{x 1} u_{x 1}$

while the total dynamic profitability in period $j\left(D_{f j}\right)$, which is a ratio of the current total profitability to the base period total profitability, is given as:

$D P_{f j}=\frac{\sum_{x=1}^{n} t_{x j} C_{x j}}{\sum_{x=1}^{n} t_{x j} u_{x j}} / \frac{\sum_{x=1}^{n} t_{x 1} c_{x 1}}{\sum_{x=1}^{n} t_{x 1} u_{x 1}}$

This value is for the total resource.

The partial dynamic profitability, $\mathrm{DP}_{\mathrm{f} x \mathrm{j}}$ of output $\mathrm{x}$ is a ratio of the current partial to the base period partial profitability, and is given as:

$D_{P_{x j}} \frac{\sum_{x=1}^{n} t_{x j} C_{x j}}{\left[t_{x j} x u_{x j}\right]} / \frac{\sum_{x=1}^{n} t_{x} 1 C_{x} 1}{\left[t_{x 1} x u_{x 1}\right]}$

This value needs to be for each resource. 
Similarly, define the total productivity in period 1 as $P_{d 1}$, which is a ratio of the current total to the base period total productivity, thus:

$$
D P_{d}=\frac{\sum_{x=1}^{n} t_{x j} C_{x 1}}{\sum_{x=1}^{n} t_{x j} u_{x 1}} / \frac{\sum_{x=1}^{n} t_{x 1} c_{x 1}}{\sum_{x=1}^{n} t_{x 1} u_{x 1}}
$$

This value is for the total resource.

The partial dynamic productivity of output $\mathrm{x}, \mathrm{DP}_{\mathrm{dx}}$, is given as:

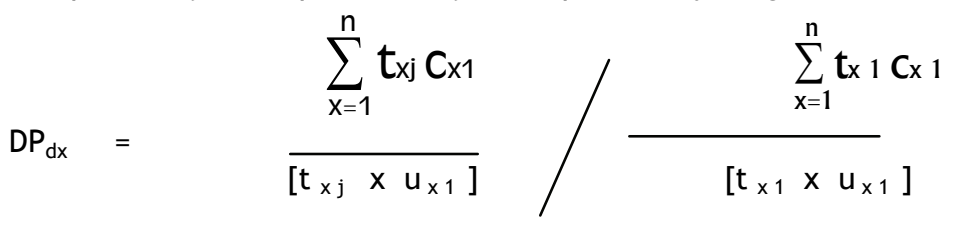

This value needs to be computed for each resource.

Also, the total dynamic price recovery factor $\left(\mathrm{DP}_{\mathrm{r}}\right)$ is given as:

$D P_{r}=\frac{\sum_{x=1}^{n} t_{x j} c_{x j}}{\sum_{x=1}^{n} t_{x j} u_{x j}} / \frac{\sum_{x=1}^{n} t_{x j} c_{x} 1}{\sum_{x=1}^{n} t_{x j} u_{x 1}}$

This value is for the total resource.

The partial dynamic price recovery factor for output $x,\left(D_{r_{x}}\right)$, is given as;

$D P_{r x}=\frac{\sum_{x=1}^{n} t_{x j} C_{x j}}{\left[t_{x j} x u_{x j}\right]} / \frac{\sum_{x=1}^{n} t_{x j} C_{x 1}}{\left[t_{x j} x u_{x 1}\right]}$

This value needs to be computed for each resource.

\subsection{Dynamic effect due to profitability}

Recall that the total dynamic profitability in period $\mathrm{j}$ is given by:

$D P_{f j}=\frac{\sum_{x=1}^{n} t_{x j} C_{x j}}{\sum_{x=1}^{n} t_{x j} u_{x j}} / \frac{\sum_{x=1}^{n} t_{x} 1 C_{x} 1}{\sum_{x=1}^{n} t_{x 1} u_{x 1}}$

Rearranging equation (5), we obtain 
$D P_{f j}=\frac{\sum_{x=1}^{n} t_{x j} C_{x j}}{\sum_{x=1}^{n} t_{x j} u_{x j}} / \frac{\sum_{x=1}^{n} t_{x 1} u_{x 1}}{\sum_{x=1}^{n} t_{x} 1 c_{x} 1}$

There are three scenarios of the values of $\mathrm{DP}_{\mathrm{f}}$ :

(i) $\mathrm{DP}_{\mathrm{f}_{\mathrm{j}}}<1$ - this implies a poor performance.

(ii) $\mathrm{DP}_{\mathrm{fj}}=1$ - this implies no change.

(iii) $\mathrm{DP}_{\mathrm{f}}>1$ - this implies a good performance.

When $\mathrm{DP}_{\mathrm{fj}}=1$, at standard operation, then

$D_{f j}=\frac{\left[\sum_{x=1}^{n} t_{x j} C_{x j} x \sum_{x=1}^{n} t_{x 1} u_{x 1}\right]}{\sum_{x=1}^{n} t_{x j} u_{x j} x \sum_{x=1}^{n} t_{x 1} C_{x 1}}=1$

Rearranging (12) gives

$\sum_{x=1}^{n} t_{x j} u_{x j}=\frac{\left[\sum_{x=1}^{n} t_{x j} C_{x j} x \sum_{x=1}^{n} t_{x 1} u_{x 1}\right]}{\sum_{x=1}^{n} t_{x 1} C_{x 1}}$

But gain (or loss) defined as $G_{f}$ is given by the difference between the standard cost and the actual cost - i.e.

$G_{f j}=\frac{\left[\sum_{x=1}^{n} t_{x j} C_{x j} x \sum_{x=1}^{n} t_{x 1} u_{x 1}\right]}{\sum_{x=1}^{n} t_{x 1} C_{x 1}}-\sum_{x=1}^{n} t_{x j} U_{x j}$

Multiplying (14) by: $\sum_{x=1}^{n} t_{x 1} u_{x 1} / \sum_{x=1}^{n} t_{x 1} u_{x 1}$,

$G_{f j}=\left(\begin{array}{cc}\frac{\sum_{x=1}^{n} t_{x j} C_{x j}}{\sum_{x=1}^{n} t_{x 1} C_{x 1}} & \frac{\sum_{x=1}^{n} t_{x j} u_{x j}}{\sum_{x=1}^{n} t_{x 1} u_{x 1}}\end{array}\right) \times \sum_{x=1}^{n} t_{x 1} U_{x 1}$

For the total output in period $j$, this represents the total gain (or loss) $\mathrm{G}_{\mathrm{f} j}$.

Note that equation (15) can also be written thus:

$G_{f j}=\left[\frac{\sum_{x=1}^{n} t_{x j} C_{x j}}{\sum_{x=1}^{n} t_{x 1} C_{x 1}} / \frac{\sum_{x=1}^{n} t_{x j} u_{x j}}{\sum_{x=1}^{n} t_{x 1} u_{x 1}}-1\right] x \sum_{x=1}^{n} t_{x j} u_{x j}$

$=\left[D P_{f j}-1\right] \times \sum_{x=1}^{n} t_{x j} u_{x j}$ 
and for partial gain it is given by:

$$
\begin{aligned}
G_{f x j} & =\left\{\frac{\sum_{x=1}^{n} t_{x j} C_{x j}}{\sum_{x=1}^{n} t_{x 1} C_{x 1}} \times \frac{t_{x 1} u_{x 1}}{t_{x j} u_{x j}}-1\right\} \times\left\{t_{x 1} \times u_{x 1}\right\} \\
G_{f x j} & =\left\{\frac{\sum_{x=1}^{n} t_{x j} C_{x j}}{\sum_{x=1}^{n} t_{x 1} C_{x 1}} \times\left[t_{x 1 j} \times u_{x 1}\right]\right\}-\left[t_{x j} \times u_{x j}\right] \\
& =\left[D P_{f j}-1\right] \times\left[t_{x j} \times u_{x j}\right]
\end{aligned}
$$

Observe that equation (17) gives the total dynamic gain or loss, $G_{f j}$, and can be expressed as:

$$
\begin{aligned}
G_{f j} & =\left[\frac{\sum_{x=1}^{n} t_{x j} C_{x j}}{\sum_{x=1}^{n} t_{x 1} C_{x 1}} t_{11} u_{11}-t_{1 j} u_{1 j}\right]+\left[\frac{\sum_{x=1}^{n} t_{x j} C_{x j}}{\sum_{x=1}^{n} t_{x 1} C_{x 1}} t_{21} u_{21}-t_{2 j} u_{2 j}\right] \\
& +\left\{\frac{\sum_{x=1}^{n} t_{x j} C_{x j}}{\sum_{x=1}^{n} t_{x 1} C_{x 1}}\left[\begin{array}{lll}
t_{n 1} & \left.\left.u_{n 1}\right]\right\}-\left[t_{n j} u_{n j}\right.
\end{array}\right]\right.
\end{aligned}
$$

An examination shows that equation (20) is the sum of the partial dynamic contributions of the individual outputs, and this represents the total dynamic contributions to profit, expressed as gain or loss (see equation 18) - i.e.

$G_{f j}=G_{f 1 j}+G_{f 2 j}+G_{f 3 j}+\ldots+G_{f n j}$

It follows from equation 21 , therefore, that the total contribution to profit is the sum of the partial contributions to profit of the individual outputs. These are for the output orientations.

\subsection{Value added element}

The value added element is related to the total contribution to profit (Agrell and Wikner [1]). We apply this concept to compute the dynamic value added due to productivity.

Recall from equation (7) that

$D P_{d}=\frac{\left[\sum_{x=1}^{n} t_{x j} C_{x 1} x \sum_{x=1}^{n} t_{x 1} u_{x 1}\right]}{\sum_{x=1}^{n} t_{x j} u_{x 1} x \sum_{x=1}^{n} t_{x 1} C_{x 1}}$

The three possibilities of change are:

(i) $\mathrm{DP}_{\mathrm{dj}}=1$ - no change.

(ii) $\mathrm{DP}_{\mathrm{dj}}>1$ - good performance.

(ii) $D_{P_{d j}}<1$ - poor performance.

When $\mathrm{DP}_{\mathrm{d}}=1$ i.e. at standard operation: 
$\sum_{x=1}^{n} t_{x j} u_{x 1}=\frac{\left[\sum_{x=1}^{n} t_{x j} C_{x 1} x \sum_{x=1}^{n} t_{x 1} u_{x 1}\right]}{\sum_{x=1}^{n} t_{x 1} C_{x 1}}$

Note that equation (22) represents the deflated cost.

Thus the total gain or loss due to dynamic productivity, $G_{d j}$, is given by:

Deflated standard cost less actual cost

Therefore:

$G_{d j}=\frac{\left[\sum_{x=1}^{n} t_{x j} C_{x 1} x \sum_{x=1}^{n} t_{x 1} u_{x 1}\right]}{\sum_{x=1}^{n} t_{x 1} C_{x 1}}-\sum_{x=1}^{n} t_{x j} U_{x j}$

Multiplying equation (23) by $\frac{\sum_{x=1}^{n} t_{x 1} u_{x 1}}{\sum_{x=1}^{n} t_{x 1} u_{x 1}}$

$\begin{aligned} G_{d j} & =\frac{\left[\sum_{x=1}^{n} t_{x j} C_{x 1} x \sum_{x=1}^{n} t_{x 1} u_{x 1}\right]}{\sum_{x=1}^{n} t_{x 1} C_{x 1}} \times \frac{\sum_{x=1}^{n} t_{x 1} u_{x 1}}{\sum_{x=1}^{n} t_{x 1} u_{x 1}}-\sum_{x=1}^{n} t_{x j} u_{x j} x \frac{\sum_{x=1}^{n} t_{x 1} u_{x 1}}{\sum_{x=1}^{n} t_{x 1} u_{x 1}} \\ & =\left(\frac{\sum_{x=1}^{n} t_{x j} C_{x 1}}{\sum_{x=1}^{n} t_{x 1} c_{x 1}}-\frac{\sum_{x=1}^{n} t_{x j} u_{x j}}{\sum_{x=1}^{n} t_{x 1} u_{x 1}} \times \sum_{x=1}^{n} t_{x 1} u_{x 1}\right.\end{aligned}$

Following logic similar to the case of total productivity, it can be shown that the value added due to partial productivity of output $x$ in period $j, G_{d x} j$, is given by:

$G_{d x j}=\left\{\left[\sum_{x=1}^{n} t_{x j} C_{x 1} / \sum_{x=1}^{n} t_{x 1} C_{x 1}\right]-\left[t_{x j} x u_{x 1}\right] /\left[t_{x 1} x u_{x 1}\right]\right\} \times\left\{t_{x 1} x_{u_{x 1}}\right\}$

\subsection{The contributions of the price recovery factor}

With respect to contribution to profit, the relationship among profitability, productivity, and price recovery factors is given as in Swaim and Sink [13], Tangen [14], Hayzen and Reeve [7], Rao [11], as:

$$
\text { Profitability }=\text { Productivity }+ \text { Price Recovery }
$$

Applying the same concept, we give the total price recovery factor $\left(P_{r j}\right)$ and the partial price recovery factor $\left(\mathrm{P}_{\mathrm{rxj}}\right)$ thus:

$P_{r j}=P_{f j}-P_{d j}$

$P_{r \times j}=P_{f \times j}-P_{d \times j}$

while the corresponding gain due to both total $\left(G_{r j}\right)$ and partial $\left(G_{r x j}\right)$ factors are expressed as:

$$
\begin{aligned}
& G_{r j}=G_{f j}-G_{d j} \\
& G_{r \times j}=G_{f \times j}-G_{d \times j}
\end{aligned}
$$


The equations (1) through (29) are used in the model that is presented below.

\section{THE INPUT AND OUTPUT-BASED PRODUCTIVITY EVALUATION MODEL}

The model developed in this work is named the Input and Output-Based Productivity Evaluation (IOP) model. The performance measures are as given in Section 2.

The format of the IOP model has 17 columns, as shown in Table 1. It has three major segments. The top segment is for the output resources at selling prices, while the middle segment is also for the output and gives the outputs performance ratios and the value added elements. This segment gives the results of the output orientation. The bottom segment is for the analysis of the inputs, and thus gives the input orientation.

\section{APPLICATION}

\subsection{Example problem}

To demonstrate the utility of the IOP model, we collected and analysed data from a soft drink bottling firm in Nigeria. This company has four outputs, while the inputs are in six categories. These are shown in Table 1 . Note that the currency used in the analysis is the Nigerian Niara.

The performance measures of Section 2 are employed for the analysis. The results are also shown in Table 1, and are discussed below.

\subsection{Results and discussion}

\subsubsection{Input orientation}

Table 2 gives the weight ratios and the changes in the measures for the inputs. The weight ratios are: labour (0.04), material $(0.89)$, energy $(0.02)$, capital $(0.04)$, and overheads $(0.01)$. Note that the weight ratio of the miscellaneous expenses reads 0.00 (in Table 2 ) owing to round-off errors. (At five decimal places, it reads 0.00253 .)

Note that materials alone, with a weight ratio of $89 \%$ of the inputs, certainly constitute a critical resource, as easily seen in the productivity result. With a partial productivity index of 2.44 (for materials alone), the overall productivity is 2.45 . This is a $145 \%$ increase in productivity, and seems satisfactory.

The total price recovery factor was very poor $(0.37)$. Note that, despite the relatively high total productivity, the very poor price recovery resulted in a combined effect of 0.91 for the firm. The results of the losses in the individual resources - i.e. labour $-53.70 \%$, materials $70.15 \%$, energy $-21.13 \%$, capital $-8.63 \%$, overheads $-21.12 \%$, and miscellaneous 928.47 - show that materials performed the worst. The implication is that costs are not fully recovered, and the intervention should focus on improving cost recovery efforts. It appears that the costing policy of the organisation is systemically faulty.

The combined effect of productivity and price recovery is 0.91 (in total profitability), which translates to a loss of 826,222.69 Naira.

\subsubsection{Output orientation:}

The results of the output orientation, the weight ratios, and the changes in the measures are shown in Table 3. 


\begin{tabular}{|c|c|c|c|c|c|c|c|c|c|c|c|c|c|c|c|c|c|c|c|c|c|c|c|c|c|c|c|c|c|c|c|c|c|}
\hline & $\begin{array}{l}n \\
\vdots \\
0 \\
0\end{array}$ & 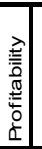 & & & & & & & 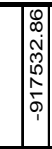 & 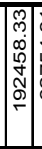 & & 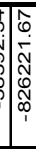 & & $\begin{array}{l} \\
0 \\
0 \\
\delta \\
\hat{0} \\
0 \\
0 \\
\end{array}$ & $\begin{array}{l}0 \\
0 \\
0 \\
0 \\
0 \\
0 \\
T \\
1\end{array}$ & & 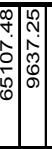 & & 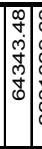 & & $\begin{array}{l}\bar{N} \\
\grave{\hat{N}} \\
\bar{\delta} \\
\bar{\delta} \\
\sim\end{array}$ & & 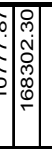 & & 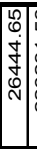 & 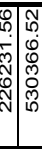 & & 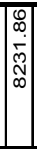 & & & & 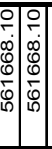 & 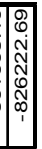 \\
\hline & 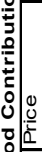 & 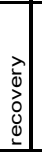 & & & & & & & 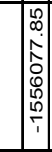 & $\mid$\begin{tabular}{l}
$\infty$ \\
\hdashline \\
$N$ \\
\hdashline \\
0 \\
0 \\
1 \\
\end{tabular} & & & & 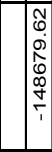 & 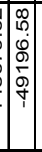 & & 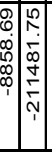 & & 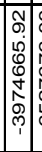 & & $\begin{array}{l}\infty \\
0 \\
1 \\
0 \\
0 \\
0 \\
0 \\
1 \\
1\end{array}$ & 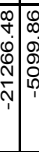 & 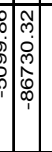 & 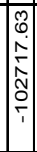 & 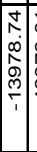 & 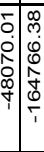 & & $\left|\begin{array}{l}0 \\
0 \\
0 \\
0\end{array}\right|$ & & & & 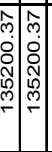 & 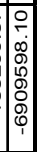 \\
\hline & 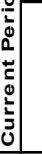 & 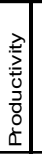 & & & & & & & \begin{tabular}{|l|} 
\\
0 \\
$\dot{j}$ \\
0 \\
0 \\
0 \\
0 \\
\end{tabular} & $\begin{array}{l}5 \\
0 \\
0 \\
0 \\
0 \\
0 \\
2 \\
N\end{array}$ & & & & 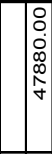 & 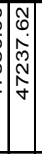 & 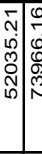 & 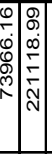 & & 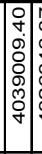 & & \begin{tabular}{l} 
\\
0 \\
$i$ \\
$\infty$ \\
0 \\
0 \\
0 \\
\hdashline \\
\end{tabular} & 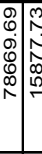 & 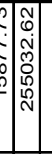 & 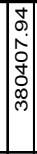 & 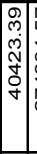 & 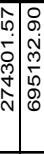 & & 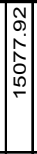 & & & & 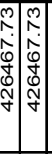 & 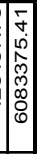 \\
\hline & & $a^{-}$ & & & & & & & \begin{tabular}{|l|} 
\\
$\infty$ \\
$\infty$ \\
0 \\
0 \\
\end{tabular} & $\mid$\begin{tabular}{l}
$\infty$ \\
$\infty$ \\
\hdashline \\
$m$
\end{tabular} & & $\begin{array}{l}0 \\
\vdots \\
\vdots \\
\vdots \\
0\end{array}$ & & \begin{tabular}{|l|}
$\infty$ \\
0 \\
0 \\
0 \\
\end{tabular} & \begin{tabular}{|c|} 
\\
$\hat{\alpha}$ \\
$\vdots$ \\
0
\end{tabular} & 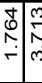 & 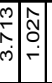 & $\begin{array}{l}\infty \\
\stackrel{\infty}{+} \\
\dot{0} \\
\end{array}$ & \begin{tabular}{c}
$N$ \\
\hdashline \\
\hdashline \\
\hdashline
\end{tabular} & & \begin{tabular}{|c|c} 
\\
$\infty$ \\
$\infty$ \\
\hdashline \\
\end{tabular} & 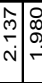 & 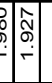 & $\begin{array}{l}\overline{\hat{N}} \\
\mathrm{~m} \\
\mathrm{~N}\end{array}$ & \begin{tabular}{c}
\multirow{2}{*}{} \\
$\bar{\sigma}$ \\
$\dot{\sigma}$ \\
\\
\end{tabular} & 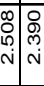 & & 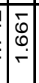 & 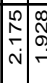 & & & 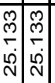 & $\begin{array}{ll}0 \\
\vdots \\
\vdots \\
0 \\
0\end{array}$ \\
\hline & & $a^{-2}$ & & & & & & & $\begin{array}{c}\bar{N} \\
\infty \\
0 \\
0\end{array}$ & 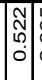 & \begin{tabular}{l|l} 
& 0 \\
0 & 0 \\
0 & $N$ \\
& 0 \\
\end{tabular} & 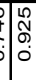 & & 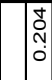 & 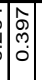 & 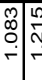 & 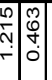 & $\begin{array}{l}\bar{\delta} \\
\dot{0} \\
0\end{array}$ & $\begin{array}{c} \\
\\
\vdots \\
0\end{array}$ & & \begin{tabular}{c|c}
5 \\
0 \\
0 \\
0
\end{tabular} & \begin{tabular}{l|l}
\multirow{2}{*}{} & 0 \\
$\infty$ & 0 \\
0 & 0 \\
0
\end{tabular} & \begin{tabular}{c|c}
0 \\
0 \\
0 \\
0
\end{tabular} & $\left|\begin{array}{l}0 \\
0 \\
0 \\
0 \\
0\end{array}\right|$ & $\begin{array}{l}N \\
0 \\
0 \\
0 \\
0 \\
\end{array}$ & 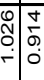 & & $\begin{array}{l}0 \\
0 \\
0 \\
0 \\
0\end{array}$ & & & & 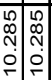 & 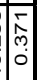 \\
\hline & & $0^{\circ}$ & & & & & & & \begin{tabular}{|l|}
\multicolumn{2}{|c|}{} \\
$\hat{0}$ \\
$\dot{-1}$
\end{tabular} & $\mid \begin{array}{l}9 \\
0 \\
0 \\
0\end{array}$ & 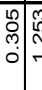 & & & 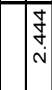 & 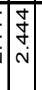 & 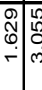 & 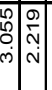 & $\begin{array}{l}\text { J } \\
\stackrel{5}{+} \\
\text { N }\end{array}$ & $\begin{array}{l}\vdots \\
⿱ \\
d \\
\end{array}$ & & 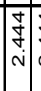 & 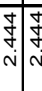 & 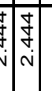 & $\begin{array}{l}\mathrm{m} \\
\mathrm{S} \\
\mathrm{N} \\
\mathrm{N}\end{array}$ & 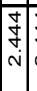 & 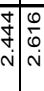 & & ì & & & & 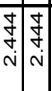 & 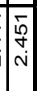 \\
\hline & : & & $\begin{array}{l}> \\
> \\
> \\
>\end{array}$ & & & & & & 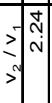 & $\mid \begin{array}{l}1 \\
0 \\
0 \\
0\end{array}$ & & & & 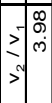 & 焉 & & 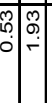 & & 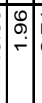 & & $\begin{array}{l}\infty \\
0 \\
\dot{r}\end{array}$ & 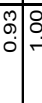 & 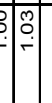 & $\mid \begin{array}{l}\infty \\
0 \\
0\end{array}$ & 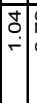 & & & $\vec{\square}$ & & & & \begin{tabular}{l|l|} 
& $\infty$ \\
0 & 0 \\
$\vdots$ & 0 \\
0
\end{tabular} & $\begin{array}{l}\infty \\
\vdots \\
\bar{N}\end{array}$ \\
\hline & & & $\begin{array}{c}0 \\
0 \\
0 \\
0\end{array}$ & 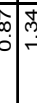 & & & \begin{tabular}{c|}
$\vec{\infty}$ \\
0 \\
0
\end{tabular} & & 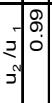 & 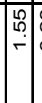 & 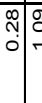 & {$\left[\begin{array}{l}\infty \\
\infty \\
0 \\
0\end{array}\right.$} & & 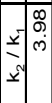 & 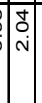 & \begin{tabular}{l|l}
$\hat{L}$ & $\hat{0}$ \\
0 & 0
\end{tabular} & 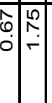 & & \begin{tabular}{l} 
\\
\hdashline \\
\hdashline \\
\end{tabular} & & $\begin{array}{l}\infty \\
\\
\\
\end{array}$ & 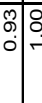 & : & 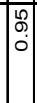 & $\begin{array}{l}\dot{y} \\
\dot{r} \\
\dot{r}\end{array}$ & \begin{tabular}{l|l}
\multirow{2}{*}{} & \multicolumn{2}{c}{} \\
0 & $\infty$ \\
0
\end{tabular} & & $\begin{array}{l}9 \\
\ulcorner \\
-\end{array}$ & \begin{tabular}{|l|l}
$\bar{\sigma}$ \\
$\bar{\sigma}$ \\
$\dot{\gamma}$
\end{tabular} & & & \begin{tabular}{l|l} 
& $\infty$ \\
0 & 0 \\
0 & 0 \\
0
\end{tabular} & $\begin{array}{l}\infty \\
\vdots \\
\bar{N}\end{array}$ \\
\hline & & & & & & & $\bar{f}$ & & $\underbrace{N} \begin{array}{l}\hat{N} \\
N\end{array}$ & $\begin{array}{l} \\
\\
0 \\
0\end{array}$ & \begin{tabular}{l|l} 
\\
\\
$\infty$ & 0 \\
$\infty$ & \\
\end{tabular} & 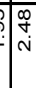 & & 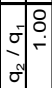 & : & 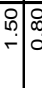 & & & $\begin{array}{l} \\
\\
-\end{array}$ & & ০a & \begin{tabular}{l|l}
8 \\
\hdashline \\
\end{tabular} & 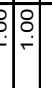 & $\begin{array}{l}\infty \\
0 \\
0 \\
0 \\
\end{array}$ & $\begin{array}{l} \\
\\
\end{array}$ & 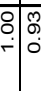 & & \begin{tabular}{|l|} 
\\
\\
\hdashline \\
\end{tabular} & $\stackrel{\circ}{\circ}$ & & & \begin{tabular}{l|l} 
\\
\\
\hdashline
\end{tabular} & ?ִ \\
\hline & & $\begin{array}{l}\frac{v}{0} \\
\frac{0}{\tilde{N}} \\
>\end{array}$ & 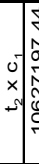 & 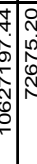 & 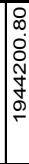 & & $\mathscr{\infty}$ & & 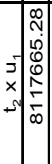 & $\mid \begin{array}{l}0 \\
\infty \\
0 \\
0 \\
0 \\
0 \\
0 \\
1\end{array}$ & 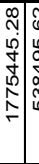 & & & 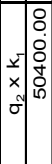 & $\begin{array}{l}8 \\
0 \\
\dot{N} \\
N \\
N \\
n\end{array}$ & 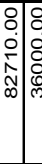 & 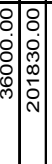 & & \begin{tabular}{|l|}
0 \\
0 \\
0 \\
2 \\
0 \\
0 \\
2 \\
2 \\
\end{tabular} & & 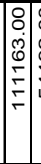 & 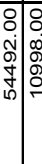 & 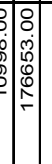 & 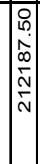 & 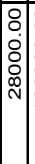 & 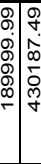 & 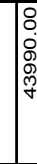 & 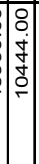 & & & & 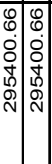 & 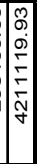 \\
\hline & $\begin{array}{l}0 \\
0 \\
\vec{\beta}\end{array}$ & \begin{tabular}{c|c}
$\frac{0}{2}$ \\
$\frac{2}{\sigma}$ \\
$>$ \\
0 \\
$\frac{\varrho}{\sigma}$ \\
0
\end{tabular} & 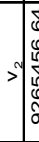 & 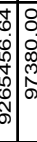 & 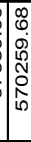 & 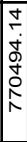 & \begin{tabular}{|l|}
0 \\
0 \\
0 \\
0
\end{tabular} & $\mid \begin{array}{l}\mid \\
\overrightarrow{0} \\
0 \\
0 \\
0\end{array}$ & 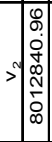 & 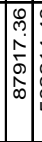 & 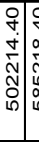 & 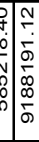 & & 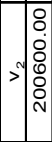 & $\begin{array}{l}8 \\
0 \\
0 \\
h \\
0 \\
0 \\
0\end{array}$ & 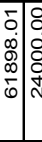 & 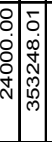 & 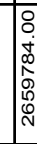 & 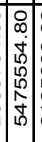 & 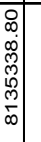 & 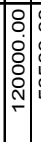 & 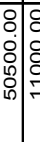 & 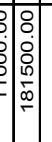 & 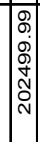 & 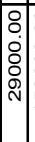 & 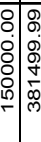 & 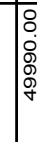 & 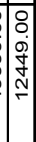 & & & & 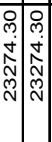 & 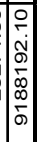 \\
\hline $\begin{array}{c}\overline{0} \\
\vdots \\
\vdots \\
\varepsilon\end{array}$ & $\begin{array}{l}0 \\
\frac{a}{3} \\
\frac{a}{\alpha} \\
\frac{\alpha}{s} \\
0\end{array}$ & $\begin{array}{l}0 \\
\frac{0}{0} \\
\frac{0}{0} \\
0\end{array}$ & ن & 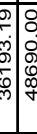 & 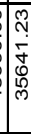 & & & $\left|\begin{array}{c|}0 \\
0 \\
0 \\
0 \\
0 \\
0\end{array}\right|$ & 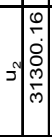 & $\mid \begin{array}{l}\infty \\
0 \\
0 \\
0 \\
0 \\
0 \\
p \\
y\end{array}$ & 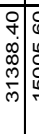 & & \begin{tabular}{|l|l}
0 \\
0.0 \\
0
\end{tabular} & 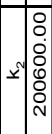 & $\begin{array}{l}8 \\
0 \\
\vdots \\
h \\
0 \\
0 \\
0\end{array}$ & 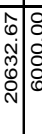 & $\begin{array}{l}8 \\
0 \\
\vdots \\
0 \\
0\end{array}$ & & 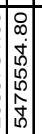 & & 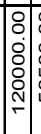 & 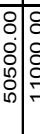 & & $\begin{array}{l}\hat{\omega} \\
\infty \\
\omega \\
N \\
\infty \\
N \\
N\end{array}$ & 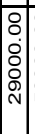 & 8 & $\overline{8}$ & $\begin{array}{l}0 \\
\infty \\
\infty \\
\infty \\
\infty\end{array}$ & 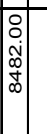 & & & 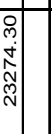 & \\
\hline $\begin{array}{l}\frac{0}{0} \\
\frac{0}{0} \\
\frac{\Xi}{\partial} \\
\partial\end{array}$ & & वे. & 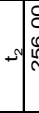 & 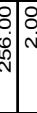 & $\begin{array}{l}8 \\
\dot{0} \\
\end{array}$ & \begin{tabular}{|l|}
8 \\
0 \\
\end{tabular} & & वे. & \begin{tabular}{r|r|} 
& 0 \\
$\dot{\varphi}$ \\
+ \\
+
\end{tabular} & $\begin{array}{l}\mathrm{D} \\
\mathrm{N}\end{array}$ & \begin{tabular}{l|l} 
& \\
$\dot{\varphi}$ & 0 \\
$\dot{0}$ & 0
\end{tabular} & & & \begin{tabular}{|c|c|c|} 
& \multirow{2}{*}{} \\
\end{tabular} & : & & & & \begin{tabular}{l} 
\\
\hdashline \\
-
\end{tabular} & & 文 & : & & $\begin{array}{l}8 \\
\vdots \\
i\end{array}$ & \begin{tabular}{|l|}
8 \\
\\
\end{tabular} & $\begin{array}{l} \\
\dot{m}\end{array}$ & 8 & $\mid \begin{array}{l}0 \\
\dot{\circ}\end{array}$ & \begin{tabular}{|l|} 
\\
\\
0
\end{tabular} & & & $\begin{array}{l} \\
\\
\end{array}$ & \\
\hline 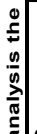 & & \begin{tabular}{c|c}
0 \\
$\frac{0}{\sigma}$ \\
$\frac{1}{0}$ \\
0 \\
$\frac{0}{\sigma}$ \\
$\infty$
\end{tabular} & $>>$ & 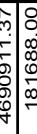 & 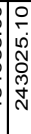 & & & $\left|\begin{array}{c}\mid \\
\vdots \\
0 \\
0 \\
0\end{array}\right|$ & 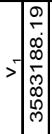 & 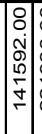 & 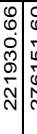 & & $\mid \begin{array}{l}\overrightarrow{0} \\
0 \\
0\end{array}$ & 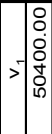 & $\begin{array}{l}0 \\
0 \\
\dot{d} \\
N \\
N \\
n\end{array}$ & 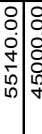 & 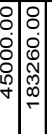 & 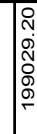 & 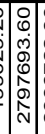 & & 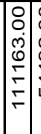 & 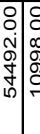 & 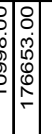 & 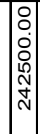 & \begin{tabular}{|l|}
8 \\
0 \\
0 \\
0 \\
0 \\
0
\end{tabular} & 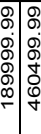 & g. & 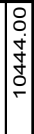 & & & & 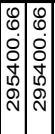 & 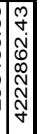 \\
\hline $\begin{array}{c}0 \\
0 \\
2 \\
2 \\
0 \\
0 \\
0 \\
0 \\
0 \\
0 \\
0 \\
0\end{array}$ & & $\begin{array}{l}0 \\
0 \\
\vdots \\
\vdots \\
0 \\
0\end{array}$ & 0. & 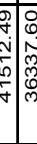 & 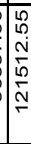 & & & $\left|\begin{array}{c}0 \\
0 \\
0 \\
0 \\
0 \\
0\end{array}\right|=$ & 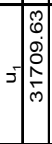 & 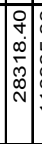 & 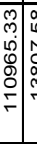 & & $\mathbb{0}$ & 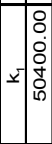 & $\begin{array}{l}0 \\
0 \\
\dot{d} \\
N \\
N \\
n\end{array}$ & 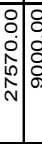 & $\begin{array}{l} \\
0 \\
0 \\
8 \\
8\end{array}$ & & 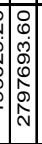 & & 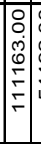 & 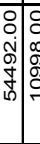 & & 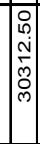 & 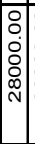 & ल्m & & \begin{tabular}{|l|}
0 \\
0 \\
$\infty$ \\
$\infty$ \\
0 \\
\end{tabular} & & & & $\begin{array}{l} \\
\\
0 \\
\dot{0} \\
\vdots \\
\vdots \\
\vdots \\
\end{array}$ & \\
\hline 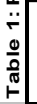 & & वे & 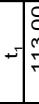 & 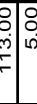 & & & & $\vec{\partial}$ & 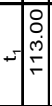 & $\mid \begin{array}{l}0 \\
0 \\
0\end{array}$ & : & & & ने & : & & & & ?ִ & & : & & & \begin{tabular}{|l|} 
\\
0 \\
$\infty$ \\
$\infty$
\end{tabular} & \begin{tabular}{|l|} 
\\
\\
\end{tabular} & 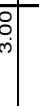 & & & \begin{tabular}{|l|} 
\\
\\
$\dot{0}$
\end{tabular} & o & & \begin{tabular}{|l|} 
\\
\\
\\
\end{tabular} & \\
\hline & 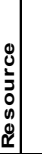 & $\begin{array}{l}0 \\
0 \\
0 \\
0 \\
0 \\
0 \\
0 \\
0\end{array}$ & & 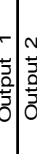 & & & & & 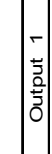 & $\mid \begin{array}{l}N \\
\vdots \\
\vdots \\
0 \\
0 \\
0\end{array}$ & & \pm & & 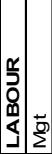 & 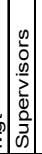 & لَّ & 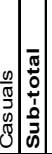 & $\begin{array}{l}0 \\
\stackrel{0}{0}\end{array}$ & 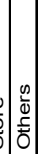 & 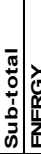 & \begin{tabular}{|l}
$\bar{\Phi}$ \\
0 \\
$\mathscr{\omega}$ \\
$\bar{\Delta}$
\end{tabular} & 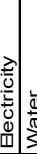 & 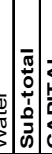 & . & $\frac{10}{0}$ & & $\frac{1}{6}$ & 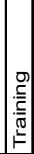 & 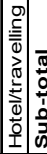 & 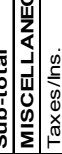 & 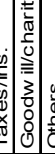 & 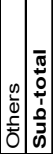 & $\mid \begin{array}{l}5 \\
0 \\
0 \\
2 \\
- \\
\vdots \\
0 \\
0 \\
1\end{array}$ \\
\hline
\end{tabular}

Table 1: Product analysis using the IOP model 
Performance Ratios and Contributions to Profit

\begin{tabular}{ccccccccc}
\multicolumn{7}{c}{ Productivity } & \multicolumn{7}{c}{ Price recovery } & \multicolumn{3}{c}{ Profitability } \\
Index & $\%$ & Contributions & Index & $\%$ & Contributions & Index & $\%$ & Contributions \\
& change & & & change & & & Change & \\
2.22 & 122.1 & 221118.99 & 0.5 & -54.33 & -211481.75 & 1.03 & 3.28 & 9637.25 \\
2.44 & 144.4 & 4326346.37 & 0.3 & -70.15 & -6527676 & 0.73 & -27.06 & -2201329.63 \\
2.44 & 144.4 & 255032.62 & 0.8 & -21.13 & -86730.32 & 1.93 & 92.73 & 168302.3 \\
2.62 & 161.6 & 695132.9 & 0.9 & -8.63 & -164766.38 & 2.39 & 139.02 & 530366.52 \\
2.44 & 144.4 & 159276.79 & 0.8 & -21.12 & -54144.02 & 1.93 & 92.77 & 105132.78 \\
2.44 & 144.4 & 426467.73 & 10 & 928.5 & 135200.37 & 25.1 & 2413.3 & 561668.1 \\
2.45 & 145.1 & 6083375.41 & 0.4 & -62.86 & -6909598.1 & 0.91 & -8.99 & -826222.69
\end{tabular}

Table 2: Weight ratios and changes in input measures

An examination of Table 3 shows the weight ratios as $0.87,0.01,0.05$, and 0.06 for outputs 1 , 2,3 , and 4 respectively. The implication is that $87 \%$ of the resources is committed to product 1 , and so it should receive a corresponding level of attention and monitoring in order to achieve the desired results.

With respect to productivity, the results vary between 0.31 and 6.11 for outputs $1,2,3$, and 4 , while the total productivity is 0.98 . Note that only output 3 recorded a loss $(-69 \%)$ in dynamic productivity; however, the overall $-1.61 \%$ loss translates to a loss of $168,865.49$ Naira. The management of output 3 therefore needs intervention.

A further examination of the results in Table 3 shows that the total price recovery $(0.92)$ was not satisfactory. A closer study of the individual outputs (Table 3 ) shows that the result for output 1 is $-17.91 \%$ (loss), while it is $-47.8 \%$ (loss), $186.47 \%$ (gain), and $-25.44 \%$ (loss) for outputs 2,3 , and 4 respectively. The overall price recovery effort of 0.92 gave a loss of $8 \%$ or $657,356.19$ Naira. Note that all except output 3 showed losses in price recovery, and require improvement.

With respect to profitability, only output 2 recorded an improvement $(219 \%)$. However, with a weight ratio of 0.01 , this did not have much effect on the total profitability, which still gave a loss of $-8.99 \%$ or $826,222.69$ Naira.

\begin{tabular}{|c|c|c|c|c|c|c|c|c|c|c|}
\hline \multicolumn{11}{|c|}{ Performance ratios and contributions to profit } \\
\hline \multirow{2}{*}{$\begin{array}{l}\text { Descrip- } \\
\text { tion } \\
\text { Output }\end{array}$} & \multirow{2}{*}{$\begin{array}{l}\text { Weight } \\
\text { Ratio }\end{array}$} & \multicolumn{3}{|c|}{ Productivity } & \multicolumn{3}{|c|}{ Price recovery } & \multicolumn{3}{|c|}{ Profitability } \\
\hline & & Index & $\begin{array}{c}\% \\
\text { change }\end{array}$ & $\begin{array}{l}\text { Contri- } \\
\text { butions }\end{array}$ & Index & $\begin{array}{c}\% \\
\text { change }\end{array}$ & $\begin{array}{l}\text { Contri- } \\
\text { butions }\end{array}$ & Index & $\begin{array}{c}\% \\
\text { change }\end{array}$ & $\begin{array}{l}\text { Contri- } \\
\text { butions }\end{array}$ \\
\hline Output 1 & 0.87 & 1.08 & 7.87 & 638544.99 & 0.8 & -17.91 & -1556077.85 & 0.89 & -11.45 & -917532.86 \\
\hline Output 2 & 0.01 & 6.11 & 510.9 & 289370.51 & 0.5 & -47.8 & -96912.18 & 3.19 & 218.91 & 192458.33 \\
\hline Output 3 & 0.05 & 0.31 & -69.5 & -1233115 & 2.9 & 186.47 & 1170360.17 & 0.88 & -12.5 & -62754.81 \\
\hline Output 4 & 0.06 & 1.25 & 25.32 & 136333.99 & 0.8 & -25.44 & -174726.32 & 0.93 & -6.56 & -38392.34 \\
\hline Total & 1 & 0.98 & -1.61 & -168865.49 & 0.9 & -7.5 & -657356.19 & 0.91 & -8.99 & -826221.67 \\
\hline
\end{tabular}

Table 3: Weight ratios and changes in output measures 
In effect, therefore, the results of the output orientation show a lag of $-2 \%$ and $-8 \%$ in productivity and price recovery respectively, and intervention is necessary.

In summary, the results of the output orientation show gaps (losses) of $-2 \%$ and $-8 \%$ in productivity and price recovery respectively, while the corresponding results for the input orientation give an increase of $145 \%$ and a loss of $-63 \%$. This shows that the costs of the resources utilised in production are not fully recovered. Thus, despite the good productivity performance of the inputs, the costs are not adequately recovered, and productivity gain is badly eroded.

Observe that the results of both orientations show the areas where intervention is needed. In the input orientation, the poor performance in price recovery obviously calls for intervention so that costs are fully recovered. As for the products, both the price recovery and productivity need improvement to ameliorate the ultimate loss in profitability. The salient point is that both orientations not only give the area for intervention, but also quantify the gap that needs to be filled.

\subsection{Products diversification}

Note that Table 3 readily presents a comparative analysis of the performance of the products. This feature could easily be used to decide which products to drop and which new ones to introduce.

Management may therefore wish to consider phasing out some poorly performing products and diversifying into others. To give effect to this, a benchmark should be spelt out. From the analysis, those products that do not meet the standard are discontinued. In this example, ranking the products in terms of their contributions to profit gives the following order: output 2 , output 4 , output 1 , and output 3 . Also, before introducing new product lines, similar exercises could be carried out to determine the expected performance of the product. These would help management to make informed decisions about the products profile, and thus to decide which products to discontinue and which ones to introduce.

\section{CONCLUSION}

This work has presented a productivity evaluation technique called the input- and outputbased productivity (IOP) evaluation model. The model simultaneously evaluates the productivity, profitability, and price recovery of the inputs and the outputs. The approach readily shows areas that need intervention in respect of both inputs and outputs, and thus provides a basis for informed intervention in both. Furthermore, it is useful for closely monitoring resources with high weight ratios.

The differences observed from the performance results in the orientations clearly underscore the importance of assessing the productivity - and, indeed, the performance - of an organization from both the input and the output perspectives. The results of the analysis from the IOP model show the gaps in performance from different viewpoints. A stand-alone technique would not give this full picture. The IOP model can be applied in product diversification.

\section{REFERENCES}

[1] Agrell, P.J. and Wikner, J. 1996. A coherent methodology for productivity analysis employing integrated partial efficiency. International Journal of Production Economics, 46-47 (1996) 401-411.

[2] Anyaeche, C.0. 2006. The development of input, output and linear programmingbased productivity evaluation model for a manufacturing firm. PhD thesis in the Department of Industrial and Production Engineering, University of Ibadan, Ibadan. 
[3] Anyaeche, C.O. and Oluleye, A.E. 1999. Profitability and productivity evaluation: A two-phase approach. Proceedings of the Annual Conference of the Nigerian Institute of Industrial Engineers, pp 37-50.

[4] Anyaeche, C.O. and Oluleye, A.E. 2003. A multi-attribute productivity model. Nigerian Journal of Industrial and System Studies, Vol. 2, No3, pp 20-28.

[5] Chan, S.K. 2000. Sustainable productivity: Asian Productivity Center at 40. Electronic Journal.

[6] Grossman, E.S. 1993. How to measure company productivity. Ed: Christopher, W. F. et al, Productivity Press, Oregon, pp 6-1.3 - 6-21. NY: North River Press.

[7] Hayzen, A.J. and Reeve, J.M. 2000. Examining relationships in productivity. Accounting Management Accounting Quarterly, Summer 2000.

[8] National Productivity Centre, and Nigerian Institute of Industrial Engineers. 1989. A proposal for developing and testing a National Productivity Guideline.

[9] Prasad, B. 1999. A model for optimizing performance based on reliability, life-circle costs and other measurements. Production Planning and Control. Vol. 10, No.30, pp 286-300.

[10] Prokopenko, J. 1987. Productivity and management: A Practical Handbook. International Labour Office, Geneva.

[11] Rao, M.P. 2000. A simple method to link productivity to profitability. Management Accounting Quarterly, Summer 2000.

[12] Sumanth, D. 1983. Productivity engineering and management. McGraw-Hill Book Company, New York.

[13] Swaim J.C. and Sink S.D. 1983. Current developments in firm or corporate level productivity measurement and evaluation: Issues in white collar productivity. Industrial Engineering Conference Proceedings, pp 8-17, Industrial Engineering Management Press.

[14] Tangen, S. 2002. Understanding the concept of productivity. Proceedings of the $7^{\text {th }}$ Asian Pacific Industrial Engineering and Management System Conference (APIEMS 2002), Taipei. E-copy via the Internet, accessed November 2002. 


\section{APPENDIX}

Notations

The following notations are used in this work.

$q_{i j}=$ quantity of input $i$ in period $j$.

$t_{x j}=$ quantity of output $x$ in period $j$.

$c_{x j}=$ unit selling price of output $x$ in period $j$.

$k_{i j}=$ unit cost price of input $i$ in period $j$.

$u_{x j}=$ unit cost price of output $x$ in period $j$.

$P_{f i j}=$ partial profitability of input $i$ in period $j$.

$P_{d i j}=$ partial productivity of input $i$ in period $j$.

$P_{r i j}=$ partial price recovery factor of input $i$ in period $j$.

$P_{d j}=$ total productivity of the firm in period $j$.

$P_{r j}=$ total price recovery factor of the firm in period $j$.

$P_{f j}=$ total profitability of the firm in period $j$.

$E_{d i j}=$ Effect on profit due to partial productivity of input $i$ in period $j$.

$E_{r i j}=$ Effect on profit due to partial price recovery of input $i$ in period $j$.

$E_{f i j}=$ Effect on profit due to partial profitability of input $i$ in period $j$.

$\mathrm{W}_{\mathrm{ij}}=$ weight of input $\mathrm{i}$ relative to the total input of the firm in period $\mathrm{j}$.

$\mathrm{z}_{\mathrm{xj}}=$ weight of output $\mathrm{x}$ relative to the total output of the firm.

$\mathrm{P}=$ Total profit of the firm.

$P_{x j}=$ Partial profit of output $x$ in period $j$.

$\mathrm{G}_{\mathrm{dx} \mathrm{j}}=$ Effect on profit due to partial productivity of output $\mathrm{x}$ in period $\mathrm{j}$.

$G_{r x j}=$ Effect on profit due to partial price recovery of output $x$ in period $j$.

$G_{f x j}=$ Effect on profit due to partial profitability of output $x$ in period $j$.

$\mathrm{G}_{\mathrm{f}}=$ total effect on profit.

$Y_{f \times j}=$ partial profitability of output $x$ in period $j$.

$Y_{d x j}=$ partial productivity of output $x$ in period $j$.

$Y_{f \times j}=$ partial price recovery of output $x$ in period $j$.

For an $\mathrm{n}$ output, $\mathrm{m}$ input system:

$$
\begin{aligned}
& x=1,2, \ldots n \\
& i=1,2, \ldots m .
\end{aligned}
$$

Base period (BP): This is the period considered a standard with which other periods can be compared.

Current period (CP): This is the period whose productivity is being evaluated.

The base period takes the subscript 1 , while the current period takes the subscript $j$. 
http://sajie.journals.ac.za 